\title{
"Occitan'île" : Projet de création d'une île artificielle de type POMU dédiée à la protection de la ressource halieutique, à la transition énergétique et à la découverte scientifique sur la mer dans le Golfe d'Aigues-Mortes
}

\author{
Jean-Marc BEYNET ${ }^{1}$, Jean-Pierre BERTHET ${ }^{2}$
}

1. Beynet-Consult, 35 rue de Peyrouse, 30320 Marguerittes, France. beynet.consult@gmail.com

2. Jean-Pierre Berthet, 299 avenue du Brusc, 83140 Six-Fours-les Plages, France. berthetjean-pierre@wanadoo.fr

\section{Résumé :}

Le projet Occitan'île consiste en la création d'une île artificielle flottante dans le Golfe d'Aigues-Mortes. Sa finalité est d'ouvrir une porte sur la Méditerranée pour la conquérir et la démocratiser, en favorisant la découverte, le partage et la diffusion des connaissances scientifiques et innovations liées à la mer. Il s'agit d'un projet social, éducatif et intergénérationnel, destiné à sensibiliser le public à tous les bienfaits que la mer peut apporter à condition de la comprendre, de la respecter et de la protéger, afin de l'exploiter intelligemment, raisonnablement et durablement, tout en préservant les ressources halieutiques et en favorisant la transition énergétique; Cette "petite POMU" (Plateforme Offshore Multi-Usages d'une superficie de l'ordre de 1 hectare) pourrait en fait être une première brique technologique de POMU plus importantes: Elle pourrait servir de démonstrateur pour tester certains dispositifs innovants en conditions réelles au large, par exemple: Ferme aquacole pédagogique en pleine mer (AMTI); Autonomie énergétique (énergies renouvelables et stockage d'hydrogène, lorsque la technique le permettra), Assainissement autonome; Production autonome en eau potable; Compensation de l'ombre portée sur les fonds; Liaisons entre modules flottants, etc. S'agissant d'une plateforme flottante, elle est réversible, et déplaçable sur d'autres sites après 30 années d'exploitation sur le DPM.

Mots-clés :

POMU (Plateforme Offshore Multi-Usages), Aquaculture, AMTI (Aquaculture Multi Trophique Intégrée, DPM (Domaine Publique Maritime), Transition écologique et énergétique,

\section{Justification du projet}

Comme l'a souligné ATTALI (2017) dans un de ses derniers ouvrages, "La mer est un lieu d'innovation et de création de produits nouveaux très prometteurs pour l'humanité, notamment en matière de santé et d'alimentation... La mer est un immense réservoir de produits nouveaux en biotechnologie, en énergie marine et en agriculture sous- 


\section{Thème 4 - Ouvrages portuaires, offshore et de plaisance}

marine...". Dans son livre il recommande aussi "d'actualiser les connaissances des océans, en particulier dans l'éducation des enfants ; développer les études scientifiques sur la mer et créer un partage des bonnes pratiques en matière de gestion des océans". Ce sera la vocation principale de ce projet qu'il est proposé d'implanter au large des côtes d'Occitanie. Cette proposition a déjà été présentée en mars 2018, lors de la consultation publique en ligne lancée par le Ministère de la transition écologique et solidaire, dans le cadre des "Stratégies de façade maritime". Sur le site MerLittoral2030, le texte ci-après a été mis en ligne le 3/03/2018 :

"Cette île serait une vitrine pour la découverte et la diffusion des connaissances et innovations liées à la mer et aux EMR. Ce projet présente un intérêt pour la collectivité publique : scientifiques, plaisanciers, non plaisanciers, scolaires,... pour faire connaitre la mer, la démocratiser et sensibiliser les jeunes générations aux bienfaits qu'elle apporte, à condition de la respecter et de la protéger. La conception devra tenir compte de l'hydrodynamique et de l'environnement et ne pas générer d'impacts négatifs sur le littoral et les fonds, la faune et la flore sousmarine. Son implantation devra faire l'objet d'une large concertation avec tous les acteurs concernés (services de l'Etat, collectivités locales, usagers de la mer, riverains, etc.). En étant mis en ouvre de manière exemplaire, ce projet développerait une nouvelle forme de tourisme écoresponsable et de loisirs de découvertes en mer. Il contribuerait au rayonnement de la région Occitanie et même de la France en Europe."

Cette proposition a reçu un bon accueil de la part du public (nombreux votes favorables et commentaires constructifs en ligne, car, sur cette île artificielle, débarqueront des touristes écoresponsables désireux de découvrir des innovations sur la mer, utiles pour les générations futures.

Les visiteurs pourront atteindre l'Occitan'île au large avec des navettes maritimes à passagers qui partiront des ports de plaisance existants autour du Golfe d'Aigues-Mortes. Le meilleur endroit pour découvrir les ressources et bienfaits que la mer peut apporter, c'est bien en étant sur la mer elle-même. La traversée en navette maritime est souhaitable pour bien préparer la visite. Un site terrestre, même proche du littoral ne procurerait pas la même sensation. Il parait important de se détacher du littoral pour favoriser le rêve, en particulier pour les jeunes générations.

La figure 1 ci-après propose une implantation, qui bien entendu, avant réalisation du projet, devra faire l'objet d'une large concertation associant les services de l'Etat, les collectivités locales, les riverains, les usagers de la mer, etc. 


\section{XVI'̀mes Journées Nationales Génie Côtier - Génie Civil \\ Le Havre 2020}

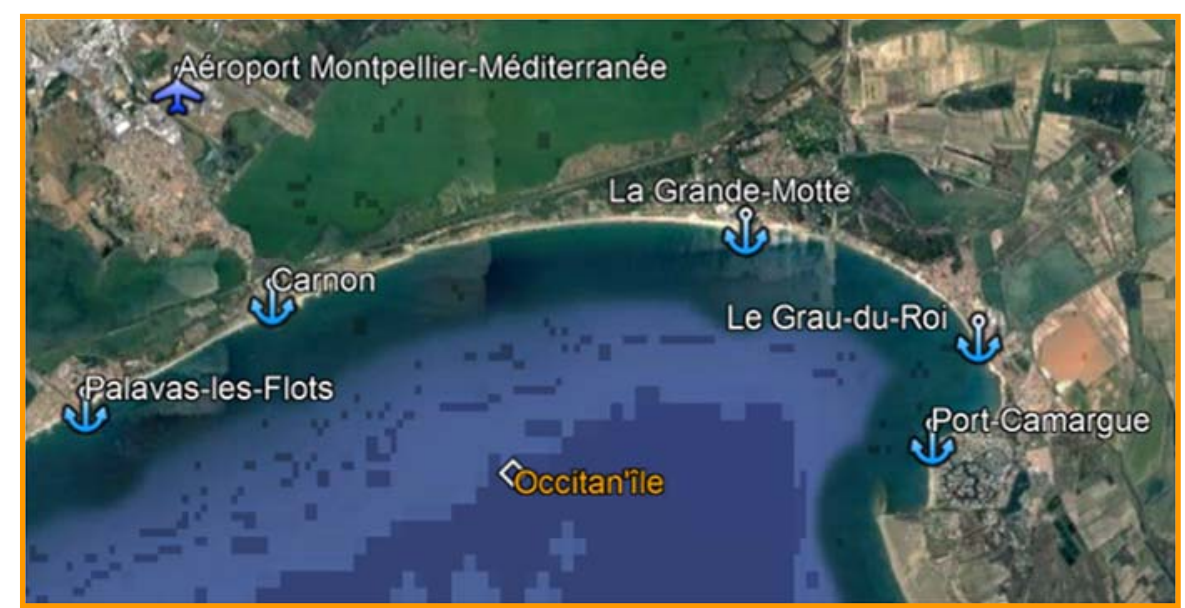

Figure 1. Implantation proposée sur fond, (source Google-Earth Pro).

Sur un flotteur $120 \mathrm{~m} \times 80 \mathrm{~m}$, d'une superficie de l'ordre de 1 hectare, il y a la possibilité d'implanter non seulement des salles pour les expositions d'innovations, séminaires d'entreprises, colloques scientifiques, ainsi que des bassins expérimentaux et pédagogiques pour l'aquaculture et la conchyliculture, mais également un restaurant pour les visiteurs et même un petit hôtel pour les congressistes ou écotouristes qui souhaiteraient rester plusieurs jours consécutifs sur l'île artificielle (figure 2). Techniquement, cette conception flottante est optimale sur ce site (flottante, donc pas d'impacts sur les fonds et facilement démontable, à moindre coût, après 30 ans d'occupation du DPM en dehors des ports existants). Elle est réversible, évolutive et exportable plus tard sur d'autres sites, en France ou en Europe.

Cette POMU flottante, dédiée à la découverte scientifique sur la mer, a été imaginée, dès l'origine, pour accueillir certaines activités liées à la production et à la transformation des ressources marines en méditerranée, tout en intégrant la conservation et la valorisation de la biodiversité marine (BEYNET, 2019).

Pour atteindre cet objectif, cette plateforme sera équipée de trois puits traversant le flotteur (figure 3). Deux puits, chacun de $1500 \mathrm{~m}^{3}$, dédiés aux expérimentations aquacoles, label pleine mer, le troisième permettant d'accéder, par plongeurs, à un volume de l'ordre d'un million de $\mathrm{m} 3$ d'eau de mer, sous le flotteur d'un hectare de surface.

- Un premier puits de $1500 \mathrm{~m}^{3}$ présentera au public une ferme aquacole expérimentale de pleine mer. Sa production restera modeste (environ 50 tonnes par an) et ne sera pas en concurrence avec la pêche professionnelle du Grau-du-Roi car elle ne représentera que $1 \%$ environ de la pêche du Grau-du-Roi, où sont débarquées en moyenne 4000 tonnes par an.

- Le second puits de $1500 \mathrm{~m}^{3}$ en pleine mer, pourra être mis à disposition des pêcheurs professionnels eux-mêmes pour accueillir des sardines en captivité trop petites pour être commercialisées et leur permettre de reconstituer rapidement leurs réserves et de rétablir leur croissance. 


\section{Thème 4 - Ouvrages portuaires, offshore et de plaisance}

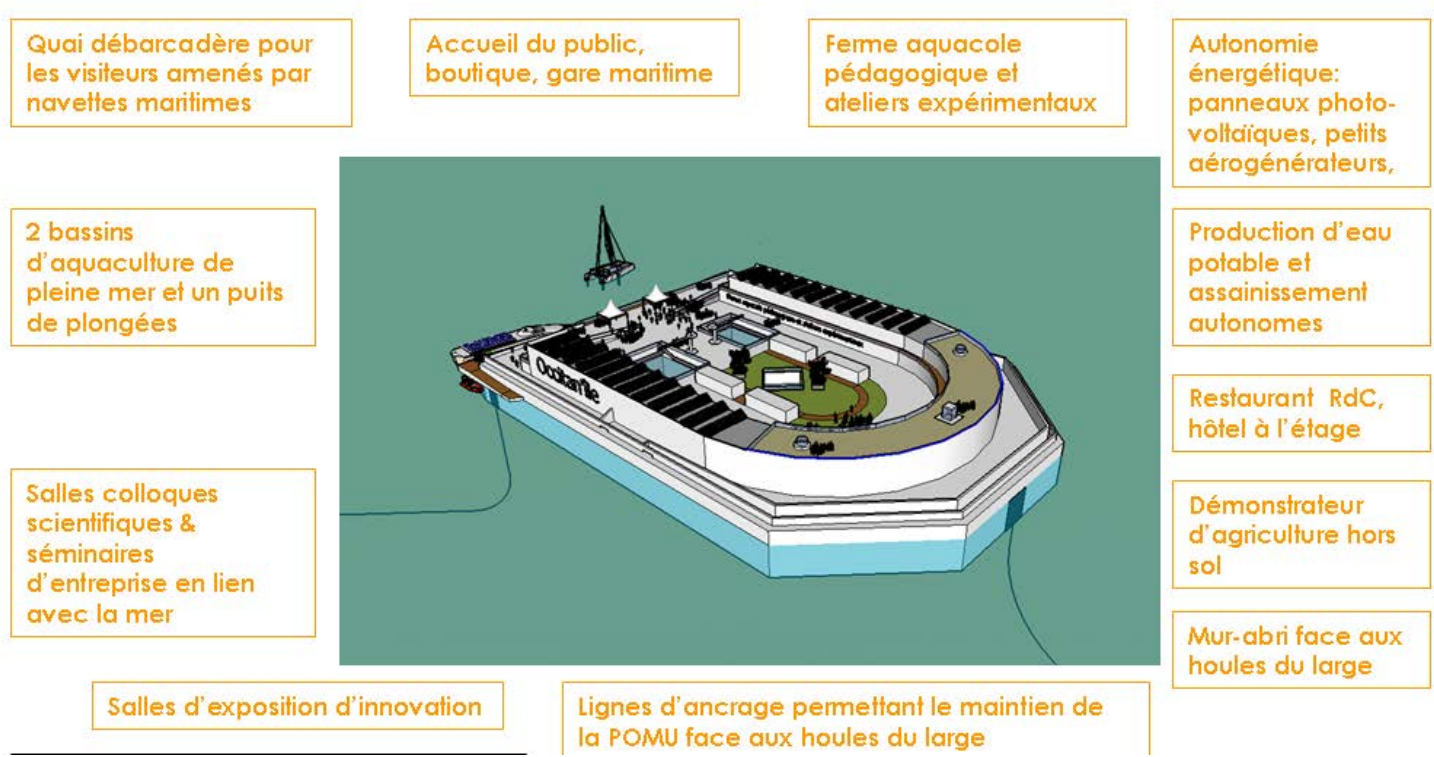

Figure 2. Localisation spatiale des différentes fonctions ou installations.
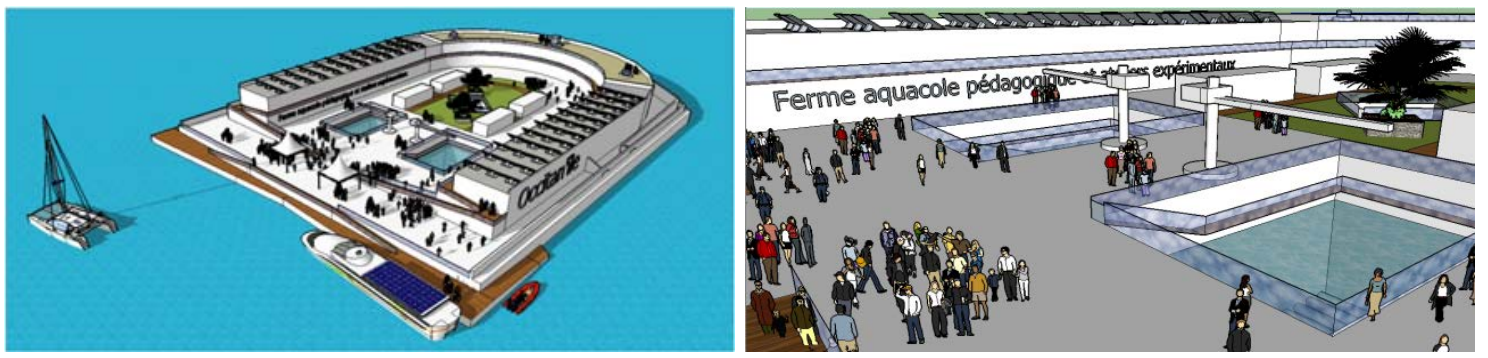

Figure 3. Accueil des visiteurs sur la plateforme offshore pour découvrir l'aquaculture de pleine mer grâce à 3 puits traversant le flotteur.

Le milieu sous-marin, sous la ferme aquacole, devient alors un atelier pour expérimenter une Aquaculture Multi Trophique Intégrée (AMTI) développée actuellement, en bassins, par les chercheurs de l'Institut Océanographique Paul Ricard, sur l'île des Embiez. Grâce au premier bassin de $1500 \mathrm{~m}^{3}$ en pleine mer, il sera possible de développer au large, l'idée de nourrir une seule espèce (daurade, loup) pour en élever plusieurs. Les autres espèces (huitres, moules, poissons planctophages et piscivores) utilisent les déchets produits par la première pour se développer. Au bout de la chaine, le fond marin se couvre d'algues qui servent de fourrage aux oursins, étoiles, concombres, vers de mer et grande nacre. De plus, l'élevage de petites moules pour filtrer et purifier l'eau de mer permettrait à d'autres espèces de se développer, d'autant plus que les petites moules, une fois récoltées, pourraient être destinées à l'alimentation animale, sous forme de farines. Ce processus sera ainsi expérimenté pour la première fois en pleine mer par les mesures, en permanence, de tous les paramètres physico-chimiques du milieu. En particulier, sera 


\section{XVI'èmes Journées Nationales Génie Côtier - Génie Civil \\ Le Havre 2020}

étudié le comportement du vivant en fonction de l'acidité, de la température, de la lumière et de l'oxygénation. L'analyse de ces données permettra d'agir au bon endroit pour rendre le cycle vertueux, sans rejets nocifs, protégeant ainsi l'équilibre de l'écosystème tout en gérant de façon économique les stocks halieutiques en amont et certains produits comestibles en aval. A ce jour, l'AMTI n'est pas parfaitement maîtrisée à terre et c'est la raison pour laquelle il est proposé un atelier-laboratoire en pleine mer.

En plus, pour garantir la biodiversité, le puits d'accès sera mis à profit pour offrir un tombant abrité où s'étageront les Biohuts ${ }^{\circledR}$. Les fonds, d'une trentaine de mètres, accueilleront des récifs-abris, offrant aux juvéniles de nouveaux habitats protecteurs. Les pêcheurs côtiers apporteront leur expérience pour vérifier l'augmentation des ressources halieutiques espérées et constater que la plateforme joue un rôle de Dispositif Concentrateur de Poissons (DCP), bien connu en Océan indien. Ils pourront ainsi profiter du stock des prédateurs qui rôdent et travailler autour, à la palangre.

Le public pourra observer, pour la première fois en France, le fonctionnement d'une ferme aquacole expérimentale de pleine mer, dont le développement maitrisé permettra en partie, à l'avenir, de nourrir l'humanité (figure 4).

Puis, au cours d'animations pédagogiques régulières, un public averti pourra voir et apprécier concrètement, dans les locaux thématiques, les résultats des travaux en cours de l'atelier sous la mer en les comparant aux prévisions.
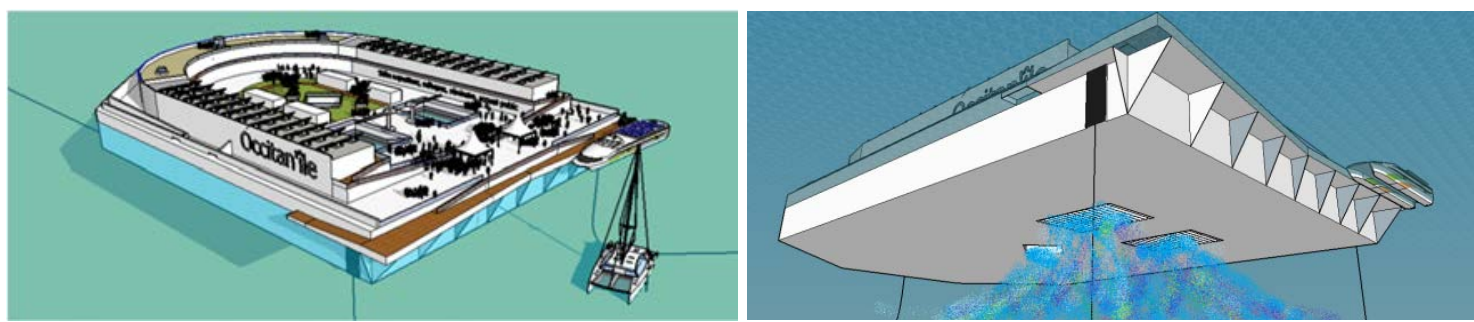

Figure 4. Vue aérienne oblique et sous-marine de la plateforme offshore.

Ce premier projet est de taille modeste (1 hectare de superficie) ce qui lui permettrait d'être réalisé par un caisson flottant monolithique unique de dimensions $120 \mathrm{~m} \times 80 \mathrm{~m} \times$ $10 \mathrm{~m}$ de haut environ. Des puits traversant sont prévus, de dimensions $12 \mathrm{~m} \times 18 \mathrm{~m}$ et permettent des expérimentations d'aquaculture en pleine mer.

Plusieurs grandes entreprises françaises et européennes disposent déjà du savoir-faire pour réaliser une telle plateforme offshore d'une seule pièce, par exemple dans la forme 10 à Marseille (la forme 10 du port de Marseille a une largeur de $85 \mathrm{~m}$ ).

Mais pour servir de démonstrateur à des projets de POMU plus importantes (on parle d'un projet flottant d'une superficie à terme de 20 ha environ au large de la Guyane), la plateforme pourrait être réalisée non pas monolithique, mais par un assemblage de 4 flotteurs $60 \mathrm{~m} \times 40 \mathrm{~m}$ (figure 5). 


\section{Thème 4 - Ouvrages portuaires, offshore et de plaisance}
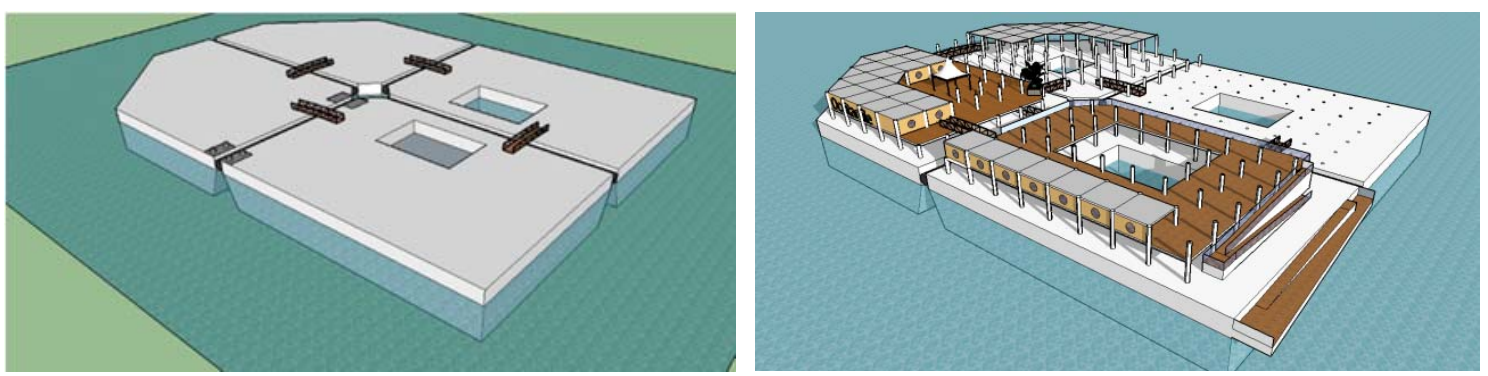

Figure 5. Exemple de conception comprenant un assemblage de quatre caissons flottants $40 \mathrm{~m} \times 60 \mathrm{~m} \times 10 \mathrm{~m}$, avec liaisons semi-élastiques.

\section{Proposition d'un programme de $R \& D$ et de mise en œuvre d'un démonstrateur}

Dans tous les cas, que le flotteur soit monolithique ou réalisé par un assemblage de 4 modules, les choix de la conception de la plateforme, des liaisons et de ses ancrages devront faire l'objet d'essais sur modèle réduit en cuve à houles. Le modèle ANEMOC (Atlas Numérique d'Etats de Mer Océaniques et Côtiers) met en évidence que dans la baie d'Aigues-Mortes, les houles dominantes du large proviennent, été comme hiver, dans le secteur $120^{\circ}-195^{\circ}$. C'est donc dans ce secteur que l'étrave de la plateforme devra être maintenue face à la houle, grâce à ses lignes d'ancrage. De plus il est important de souligner que la hauteur significative des houles hivernales est inférieure à $1 \mathrm{~m}$ pendant $70 \%$ du temps. En saison estivale, les houles du large ont une hauteur significative inférieure à $0,50 \mathrm{~m}$ pendant près de $60 \%$ du temps, et inférieure à $1 \mathrm{~m}$ pendant $30 \% \mathrm{du}$ temps. En saison estivale, les houles du large de hauteur significative comprise entre 1 et $2,5 \mathrm{~m}$ règnent pendant 10 à $12 \%$ du temps.

Si les tests en cuve à houles montrent que les risques de submersion marine sont à craindre en hiver, on pourrait imaginer de dissocier les niveaux en ajoutant une plateforme épontillée pour l'accueil du public. La plateforme dont le franc-bord serait, réglé à $3 \mathrm{~m}$ au-dessus de la surface, pourrait supporter des franchissements de vagues lors des plus fortes tempêtes hivernales. Hors épisode de tempête, ce pont à $3 \mathrm{~m}$ au-dessus de la flottaison serait dédié aux professionnels (aquaculteurs, pêcheurs, conchyliculteurs, scaphandriers). Les éleveurs de la ferme aquacole expérimentale pédagogique auraient accès aux deux puits éclairés à l'arrière ainsi qu'aux deux puits en pénombre (option). Les conchyliculteurs auraient accès à leurs filières périphériques. Les plongeurs travailleraient dans l'atelier sous-marin, à partir du puits central. Le suivi et la tenue des liaisons souples entre flotteurs (sans doute instrumentalisées) et de l'accrochage des lignes d'ancrage en serait facilité. Toujours en dehors des épisodes de tempêtes hivernales, après leur débarquement sur la plateforme offshore, les visiteurs écotouristes, seraient invités à se regrouper sur un pont supérieur calé $3 \mathrm{~m}$ plus haut. Les épontilles (pilotis) permettent une structure type jacket, soutenant un pont de circulation du public avec revêtement bois. Les épontilles de diamètre $600 \mathrm{~mm}$ environ, seraient prolongées en 


\section{XVI'̀mes Journées Nationales Génie Côtier - Génie Civil \\ Le Havre 2020}

hauteur pour servir d'ossature aux différents locaux, d'une superficie totale de 1800 à $2000 \mathrm{~m}^{2}$ environ sur le pont supérieur. Les remplissages pourraient être en bois. Les flotteurs pourraient être en béton, ou en acier.

Les liaisons semi-élastiques amorties entre plateformes pourraient être mises au point, d'abord grâce à des modélisations numériques (logiciel type Orcaflex), puis vérifiées ensuite sur modèle physique réduit en cuve à houles.

Par exemple, des défenses cylindriques continues pourraient être mise en œuvre, juste audessus de la ligne de flottaison, entre deux flotteurs en vis-à-vis. Puis les liaisons entre caissons ne devront probablement pas être rigides, mais souples au contraire afin d'absorber une partie des mouvements et efforts de la houle. A priori, ces liaisons pourraient être réalisées par des amortisseurs du type parasismique, frein dynamique, reliant les flotteurs par l'intermédiaire d'articulation ou rotules sur platines.

Si la décision est prise de construire cette plateforme Occitan'île, non pas monolithique mais par assemblage de 4 flotteurs, un programme de R\&D devra être mis en place préalablement aux études détaillées pour bien étudier ces points délicats, qui seront très utiles à la réalisation d'autres POMU de taille plus importante, dans le futur.

Dans le cas où la plateforme flottante serait réalisée par l'assemblage de 4 pontons de 40 $\mathrm{m} \times 60 \mathrm{~m}$, les superstructures devront être indépendantes sur chaque module élémentaire, le passage d'un module à l'autre étant assuré par des passerelles articulées similaires à celles qui existent sur les ports de plaisance.

\section{Conclusions}

En étant mis en œuvre de manière exemplaire dans le Golfe d'Aigues-Mortes, ce projet Occitan'île contribuerait à l'éducation du public et des jeunes générations en particulier, pour respecter la mer et préserver les ressources halieutiques ainsi que pour sensibiliser le public à la transition écologique et énergétique. Il est important de rappeler qu'il serait utile aussi pour la préservation des ressources halieutiques, dans l'intérêt de la pêche professionnelle. Cette île artificielle serait une sorte de Totem maritime au large de l'Occitanie, qui ferait écho aux aménagements terrestres en cours du Plan Littoral 21.

Bien entendu, l'Ifremer et les usagers de la mer comme les pêcheurs professionnels devront être consultés préalablement lors de concertations à venir et associés au projet, afin d'optimiser l'implantation et la conception de cette île artificielle en tenant compte de l'ensemble des contraintes. Par ailleurs, il est à noter qu'étant située assez au large (3 à 4 milles marins) avec une hauteur limitée au-dessus de la mer (de l'ordre d'une dizaine de mètres), cette île artificielle flottante ne présenterait pas d'impact visuel significatif depuis la côte.

Mais bien entendu, la mise en œuvre de ce projet est conditionnée à la remise du rapport du CGEDD (missionné par le Ministère de la Transition Ecologique et Solidaire), concernant les enjeux de sécurité, juridiques, et contractuels (taxes en particulier), ainsi 


\section{Thème 4 - Ouvrages portuaires, offshore et de plaisance}

que les enjeux de sûreté, gestion des risques, gestion des déchets et problématiques environnementales de ce type d'aménagement flottant en mer pour recevoir du public. Lorsque le rapport du CGEDD sera connu et que le nombre de visiteurs potentiels pourra être estimé en fonction des périodes (en tenant compte des conditions météorologiques, climats de houle en particulier), une étude de rentabilité économique et financière pourra être entreprise en considérant les dépenses (coûts des travaux et frais de fonctionnement) et les recettes (prix du billet visiteurs, location de salles pour séminaires d'entreprises, colloques scientifiques, etc.). Dans l'attente de pouvoir réaliser cette étude de faisabilité économique et financière, il est possible de s'appuyer sur les études approfondies qui ont pu être réalisées sur des POMU plus importantes (et notamment la POMU guyanaise) dont les conditions de faisabilité et de succès ont déjà été bien identifiés (LOCKHART \& BUDOC, 2015 ; RABAIN, 2014, 2019a, 2019b). Des ordres de grandeur de coûts peuvent être avancés malgré tout dès à présent, mais en restant prudent à ce stade très préliminaire du projet. Par exemple 15 à $20 \mathrm{M}$.HT pour le flotteur (monolithique ou non), y compris ses ancrages et 5 à $10 \mathrm{M}$.HT pour les superstructures sur épontilles, selon standing de finition des bâtiments et équipements (petits aérogénérateurs, panneaux photovoltaïques, stockage de l'énergie, désalinisation de l'eau de mer et traitement des eaux usées, etc.). Quant au programme de $R \& D$, en première approche, il pourrait correspondre à 3 ou $4 \%$ du coût du flotteur, en particulier pour les modélisations physiques en cuve à houle.

\section{Références bibliographiques}

ATTALI J. (2017). Histoires de la mer. Editions Fayard, 360p. EAN: 9782213706689 BEYNET J.-M. (2019). Proposition de création d'une île artificielle dédiée à la découverte scientifique sur la mer dans le Golfe d'Aigues-Mortes, Présentation orale au Groupe Synergie POMU (Plateforme Offshore Multi-Usages) du Cluster Maritime Français, le 12/06/2019 à Paris.

LOCKHART T., BUDOC R.-L. (2015). Des plateformes offshore multi-usages, comme moyen de développer l'économie circulaire - Présentation du concept et illustration par un cas d'application en Guyane Française. Economie circulaire et écosystèmes portuaires, pp. 381-392. Collection Les Océanides.

RABAIN A. (2014). La maritimisation de l'économie a démarré. L'Usine Nouvelle, 27 Novembre 2014. https://www.usinenouvelle.com/article/la-maritimisation-de-l-economie-a-demarre.N300048 RABAIN A. (2019a). Présentation POMU. Intervention orale lors du Groupe SynergiePlateformes Offshore Multi-Usages, Paris, 12 juin 2019.

RABAIN A. (2019b). Plateformes offshore multi-usages : Fondamentaux des POMU et potentiels de marché - Cas pratique Guyane : Caractérisation des enjeux clés. Les Assises Port du Futur, Lille, 25 septembre 2019, 15 p.

www.portdufutur.fr/sites/portdufutur/files/fichiers/2019/10/Rabain 